\title{
STABILITY ANALYSIS OF ORTHOTROPIC RECTANGULAR PLATES COMPRESSED ALL OVER THE CONTOUR
}

\author{
Andrey Victorovich Korobko* \\ Orel State University, Russia \\ Sergey Yurievich Savin \\ South-West State University, Russia \\ Ivan Andreevich Ivlev \\ Orel State University, Russia
}

The article describes the stability analysis of elastic orthotropic rectangular plates with combined boundary conditions (combination of simple supporting and clamping along the sides). The external load compresses a plate along all over the contour. Authors propose to apply the form factor interpolation method (FFIM) to calculate the critical load of buckling. The FFIM is based on the functional relationship between integral geometric parameter of the midplane such as the form factor and the critical force of the buckling. It was obtained the approximate analytical expressions for the critical force of orthotropic rectangular plates. The form factor and the flexural stiffness ratios are parameters of these approximate expressions. The calculation results are compared with the FEM solutions obtained in the program SCAD Office and demonstrate good accuracy. The proposed approach can be extended to other forms of plates, boundary condition combinations, as well as the types of loading.

Key words: Orthotropic plates, Combined boundary conditions, The form factor interpolation method, The FEM, Stability analysis, Loads, Compression

\section{INTRODUCTION}

A stability analysis is one of the main problems, which must be solved by engineers, when they design plates or thin-walled rods. In the both cases the problem can be described by the stability equation of the plate theory. Basic positions of this theory were formulated by Timoshemko S.P., Volmir A.S. [01, 02] and etc. However, there are a range of engineering problems, which require of analysis.

\section{LITERATURE REVIEW}

The variational method and the asymptotic method dominate among the analytical methods now. Dmitrienko Yu.I. [03] derived asymptotic stability equations of laminated plates theory. Author used asymptotic expansion to small geometric parameter (thickness to length ratio) row. Belous A.A. and Belous V.A. [04] analyzed buckling of rectangular plate for different variants of loads. In the paper authors described compressible of material and obtained relationships between stresses and strains. These relationships can be used to solve different stability problems of plates and shells theory.

In the paper [05] authors used the iteration method to obtain the critical load for clamped plate, which is compressed in one direction. Lopatin A.V. and Avakumov R.V. [06] used the finite element method to stability analysis of plates, two sides of which are not supported. The articles [07-09] describes local buckling of the thin wall of I - beams.

Thus, it can be concluded that almost all analytical solution are obtained for rectangular or circle plates. When it is necessary to analyze plate with other shape of the midplane it is applied numerical calculation methods which are realized in computer programs. However in this case it requires paying attention to direction of local axes of each finite element since it can be wrong if triangulation is automatical.

Therefore, the authors of this paper suggest using the form factor interpolation method (FFIM) 
analyze stability of elastic orthotropic plates. The form factor interpolation method is based on the functional relationship between the integral characteristics of the plate: geometric (form factor) and mechanical (maximum deflection, frequency of natural oscillations, critical force of buckling) [10]. It is should be noted that a form factor is a contour integral of the plate midplane:

$$
K_{f}=\min \oint_{L} \frac{d s}{h}
$$

where ds is the elementary section of the contour of the plate midplane, $\mathrm{h}$ is the perpendicular to ds from a certain point that is called the pole and should be chosen so that the value of $\mathrm{Kf}$ is minimum.

As it is shown in the paper [11] conformal radiuses ratio can be used as a geometric analog of the integral mechanical characteristics of plates too. However, this parameter is not applied to stability analysis at the moment.

A.V. Korobko and A.V. Chikulaev [12, 13] apply the FFIM to the stability analysis of shallow and spherical isotropic shells. In the papers [14-16] authors use the form factor interpolation method to the solution of dynamics and statics problems of orthotropic plates. That allows us to extend the FFIM to stability analysis of orthotropic plates. One of the main paths of the method development is obtaining of the boundary surfaces of the critical force for the basic forms of plate midplane such as rectangles, rhombs, triangles, polygons.

\section{METHODS AND MATERIALS}

In this article we present the solution of the stability problem for rectangular elastic orthotropic plates which have combined boundary conditions (combination of simple supporting and clamping along the sides) as it is shown in the figure 1 . Plates are compressed by the evenly distributed load all over the contour. We used program Table Curve 2D and 3D to obtain approximate analytical relationship between form factor, flexural rigidity ratios and critical force. Initial dates were obtained in the SCAD Office program (based on the FEM) to rectangular plates with side ratios $0.1,0.2, \ldots, 1$ and flexural rigidity ratios $\mathrm{Dx} / \mathrm{H}=1$, 2 ... 5; Dy/H = 1, $2 \ldots 5$.

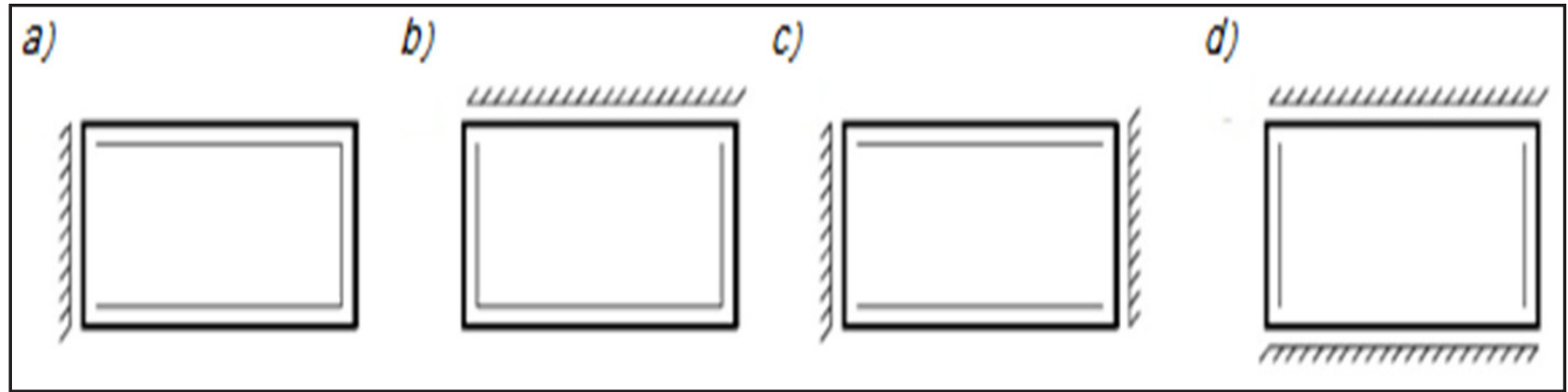

Figure 1: The variations of boundary conditions for rectangular plates

\section{RESEARCHS AND RESULTS}

Stability equation is well known from thin plates theory [17]:

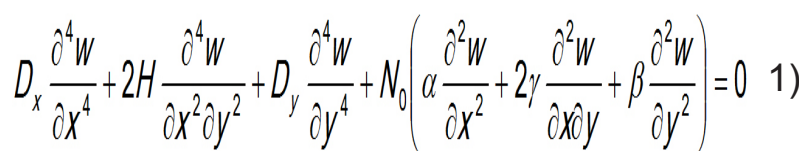

where $\mathrm{H}=\mathrm{D}_{1}+2 \mathrm{D}_{\mathrm{xy}}, \mathrm{D}_{1}=\mathrm{D}_{\mathrm{x}} \mathrm{V}_{y}=\mathrm{D}_{y} \mathrm{~V}_{x}$

No is parameter which characterize critical force of buckling; $D_{x^{\prime}}, D_{y^{\prime}} D_{x y}$ are flexural rigidities; $V_{x^{\prime}}$ $V_{y}$-Poisson's ratios; $\alpha, \gamma, \beta$ are coefficients from equalities $N x=\alpha N o, N y=\beta N o, N x y=\gamma N o(N x, N y$, $N x y$ are internal forces).
If a plate is compressed by load that evenly distributed along the contour then the stability equation takes the form:

$D_{x} \frac{\partial^{4} W}{\partial x^{4}}+2 H \frac{\partial^{4} W}{\partial x^{2} \partial y^{2}}+D_{y} \frac{\partial^{4} W}{\partial y^{4}}+N_{0} \frac{\partial^{2} W}{\partial x^{2}}+N_{0} \frac{\partial^{2} W}{\partial y^{2}}=0$

We divide equation to flexural rigidity $\mathrm{H}$ and take the follow substitution

$$
K_{n}=N \cdot \frac{A}{H} ; \quad \eta_{1}=\frac{D_{x}}{H} ; \quad \eta_{2}=\frac{D_{y}}{H}
$$


In this case we obtain the differential equation:

$$
\eta_{1} \frac{\partial^{4} w}{\partial x^{4}}+2 \frac{\partial^{4} w}{\partial x^{2} \partial y^{2}}+\eta_{2} \frac{\partial^{4} w}{\partial y^{4}}+K_{n} \frac{\partial^{2} w}{\partial x^{2}}+K_{n} \frac{\partial^{2} w}{\partial y^{2}}=0
$$

where $K n$ is function of dimensionless critical force. Using the FEM (SCAD Office program)

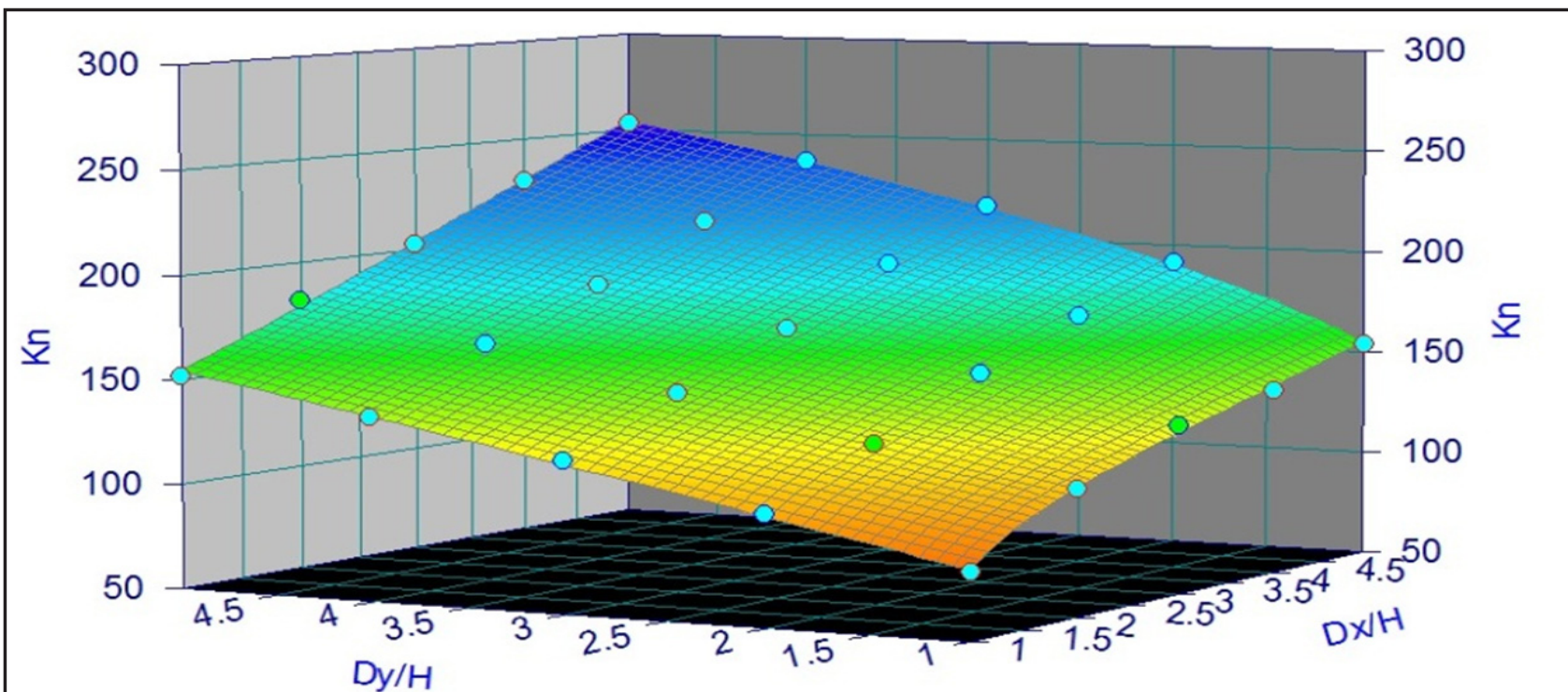

Figure 2: Diagram $\mathrm{Kn}$ - flexural rigidity ratios for a rectangular plate with side ratio $a / b=0.5$ and boundary conditions corresponding to the diagram "a" in the figure 1

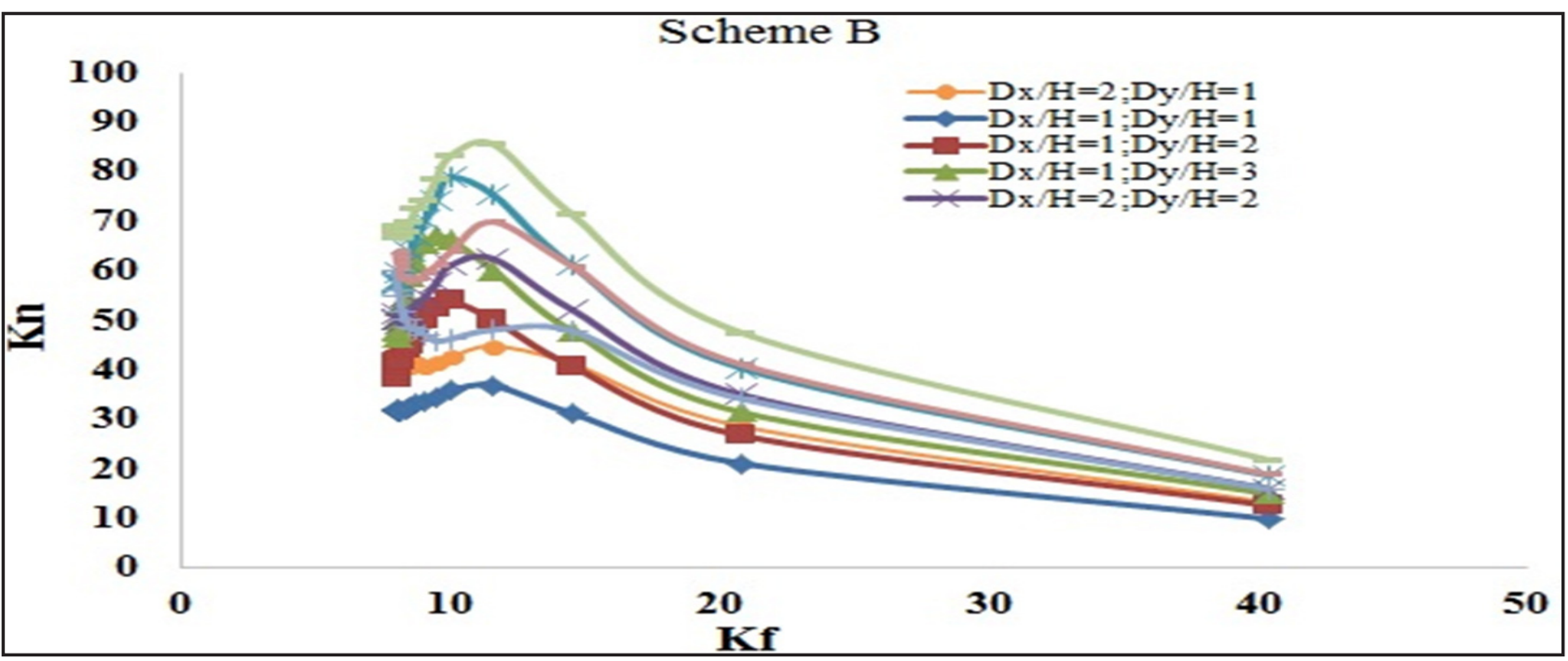

Figure 3: The graph of $K n-K f$ when boundary conditions correspond to the scheme " $b$ " in the figure 1

The graph's form in the figures 2, 3 shows that flexural rigidities ratio. Thus we find parameter there is functional relation of $\mathrm{Kn}$, form factor and $\mathrm{Kn}$ in the form

$$
K_{n}=a_{1}+a_{2} \eta_{1}+a_{3} \eta_{2}+a_{4} \eta_{1}^{2}+a_{5} \eta_{2}^{2}+a_{6} \eta_{1} \eta_{2}+a_{7} \eta_{1}^{3}+a_{8} \eta_{2}^{3}+a_{9} \eta_{1} \eta_{2}^{2}+a_{10} \eta_{1}^{2} \eta_{2}
$$

where ai is parameter which depends on a form factor [10] as it is shown in the figure 3,b.
We propose to obtain parameters of formula (4) in the following form: 


$$
a_{i}=\sum_{j=1}^{n} A_{n} K_{f}^{(1-n)}
$$

where $A n$ is coefficients that depend on the boundary conditions of a plate. An values are presented in the tables $1-4$.

Table 1: The parameters An in the formula (5) for combined boundary conditions that is shown in the Figure 1,a

\begin{tabular}{|c|c|c|c|c|c|c|c|c|c|c|}
\hline \multirow{2}{*}{ n (5) } & \multicolumn{10}{|c|}{ The parameters a in the formula (4) } \\
\hline & a1 & a2 & a3 & a4 & a5 & a6 & a7 & a8 & a9 & a10 \\
\hline 1 & $-2,15 \cdot 10^{6}$ & $-4,76 \cdot 10^{10}$ & $4,36 \cdot 10^{8}$ & $-5,09 \cdot 10^{7}$ & $-4,07 \cdot 10^{7}$ & $8,73 \cdot 10^{7}$ & $-6,28 \cdot 10^{6}$ & $6,59 \cdot 10^{5}$ & $-3,20 \cdot 10^{5}$ & $-6,31 \cdot 10^{5}$ \\
\hline 2 & $2,82 \cdot 10^{8}$ & $7,49 \cdot 10^{12}$ & $-6,52 \cdot 10^{10}$ & $7,50 \cdot 10^{9}$ & $6,17 \cdot 10^{9}$ & $-1,56 \cdot 10^{10}$ & $9,45 \cdot 10^{8}$ & $-1,10 \cdot 10^{8}$ & $4,52 \cdot 10^{7}$ & $8,88 \cdot 10^{7}$ \\
\hline 3 & $-1,54 \cdot 10^{10}$ & $-5,17 \cdot 10^{14}$ & $4,23 \cdot 10^{12}$ & $-4,77 \cdot 10^{11}$ & $-4,06 \cdot 10^{11}$ & $1,23 \cdot 10^{12}$ & $-6,17 \cdot 10^{10}$ & $7,97 \cdot 10^{9}$ & $-2,73 \cdot 10^{9}$ & $-5,34 \cdot 10^{9}$ \\
\hline 4 & $4,70 \cdot 10^{11}$ & $2,09 \cdot 10^{16}$ & $-1,59 \cdot 10^{14}$ & $1,75 \cdot 10^{13}$ & $1,55 \cdot 10^{13}$ & $-5,66 \cdot 10^{13}$ & $2,34 \cdot 10^{12}$ & $-3,34 \cdot 10^{11}$ & $9,37 \cdot 10^{10}$ & $1,83 \cdot 10^{11}$ \\
\hline 5 & $-8,86 \cdot 10^{12}$ & $-5,56 \cdot 10^{17}$ & $3,85 \cdot 10^{15}$ & $-4,15 \cdot 10^{14}$ & $-3,82 \cdot 10^{14}$ & $1,70 \cdot 10^{15}$ & $-5,72 \cdot 10^{13}$ & $9,04 \cdot 10^{12}$ & $-2,05 \cdot 10^{12}$ & $-3,97 \cdot 10^{12}$ \\
\hline 6 & $1,08 \cdot 10^{14}$ & $1,03 \cdot 10^{19}$ & $-6,38 \cdot 10^{16}$ & $6,71 \cdot 10^{15}$ & $6,45 \cdot 10^{15}$ & $-3,52 \cdot 10^{16}$ & $9,57 \cdot 10^{14}$ & $-1,66 \cdot 10^{14}$ & $2,98 \cdot 10^{13}$ & $5,75 \cdot 10^{13}$ \\
\hline 7 & $-8,53 \cdot 10^{14}$ & $-1,35 \cdot 10^{20}$ & $7,39 \cdot 10^{17}$ & $-7,56 \cdot 10^{16}$ & $-7,62 \cdot 10^{16}$ & $5,18 \cdot 10^{17}$ & $-1,12 \cdot 10^{16}$ & $2,12 \cdot 10^{15}$ & $-2,95 \cdot 10^{14}$ & $-5,66 \cdot 10^{14}$ \\
\hline 8 & $4,24 \cdot 10^{15}$ & $1,29 \cdot 10^{21}$ & $-6,01 \cdot 10^{18}$ & $5,97 \cdot 10^{17}$ & $6,31 \cdot 10^{17}$ & $-5,47 \cdot 10^{18}$ & $9,21 \cdot 10^{16}$ & $-1,89 \cdot 10^{16}$ & $1,96 \cdot 10^{15}$ & $3,74 \cdot 10^{15}$ \\
\hline 9 & $-1,20 \cdot 10^{16}$ & $-8,84 \cdot 10^{21}$ & $3,36 \cdot 10^{19}$ & $-3,24 \cdot 10^{18}$ & $-3,60 \cdot 10^{18}$ & $4,12 \cdot 10^{19}$ & $-5,22 \cdot 10^{17}$ & $1,16 \cdot 10^{17}$ & $-8,41 \cdot 10^{15}$ & $-1,59 \cdot 10^{16}$ \\
\hline 10 & $1,49 \cdot 10^{16}$ & $4,25 \cdot 10^{22}$ & $-1,24 \cdot 10^{20}$ & $1,15 \cdot 10^{19}$ & $1,35 \cdot 10^{19}$ & $-2,17 \cdot 10^{20}$ & $1,94 \cdot 10^{18}$ & $-4,62 \cdot 10^{17}$ & $2,11 \cdot 10^{16}$ & $3,96 \cdot 10^{16}$ \\
\hline 11 & & $-1,36 \cdot 10^{23}$ & $2,70 \cdot 10^{20}$ & $-2,43 \cdot 10^{19}$ & $-3,00 \cdot 10^{19}$ & $7,55 \cdot 10^{20}$ & $-4,29 \cdot 10^{18}$ & $1,09 \cdot 10^{18}$ & $-2,34 \cdot 10^{16}$ & $-4,37 \cdot 10^{16}$ \\
\hline 12 & & $2,62 \cdot 10^{23}$ & $-2,65 \cdot 10^{20}$ & $2,29 \cdot 10^{19}$ & $3,00 \cdot 10^{19}$ & $-1,57 \cdot 10^{21}$ & $4,25 \cdot 10^{18}$ & $-1,15 \cdot 10^{18}$ & & \\
\hline 13 & & $-2,28 \cdot 10^{23}$ & & & & $1,48 \cdot 10^{21}$ & & & & \\
\hline
\end{tabular}

Table 2: The parameters An in the formula (5) for combined boundary conditions that is shown in the Figure 1,b

\begin{tabular}{|c|c|c|c|c|c|c|c|c|c|c|}
\hline \multirow{2}{*}{$n(5)$} & \multicolumn{10}{|c|}{ The parameters a in the formula (4) } \\
\hline & a1 & a2 & a3 & a4 & a5 & a6 & a7 & a8 & a9 & a10 \\
\hline 1 & $-4,01 \cdot 100^{8}$ & $5,51 \cdot 10^{8}$ & $2,69 \cdot 10^{6}$ & $-1,66 \cdot 10^{8}$ & $8,81 \cdot 10^{7}$ & $-1,04 \cdot 10^{8}$ & $1,03 \cdot 10^{7}$ & $-1,13 \cdot 10^{7}$ & $-9,98 \cdot 10^{8}$ & $2,26 \cdot 10^{7}$ \\
\hline 2 & $5,98 \cdot 10^{10}$ & $-8,22 \cdot 10^{10}$ & $-2,88 \cdot 10^{8}$ & $2,47 \cdot 10^{10}$ & $-1,32 \cdot 10^{10}$ & $1,56 \cdot 10^{10}$ & $-1,53 \cdot 10^{9}$ & $1,69 \cdot 10^{9}$ & $1,57 \cdot 10^{11}$ & $-3,38 \cdot 10^{9}$ \\
\hline 3 & $-3,87 \cdot 10^{12}$ & $5,32 \cdot 10^{12}$ & $1,01 \cdot 10^{10}$ & $-1,60 \cdot 10^{12}$ & $8,58 \cdot 10^{11}$ & $-1,01 \cdot 10^{12}$ & $9,92 \cdot 10^{10}$ & $-1,10 \cdot 10^{11}$ & $-1,08 \cdot 10^{13}$ & $2,19 \cdot 10^{11}$ \\
\hline 4 & $1,44 \cdot 10^{14}$ & $-1,99 \cdot 10^{14}$ & $-1,84 \cdot 10^{10}$ & $5,98 \cdot 10^{13}$ & $-3,23 \cdot 10^{13}$ & $3,78 \cdot 10^{13}$ & $-3,71 \cdot 10^{12}$ & $4,14 \cdot 10^{12}$ & $4,39 \cdot 10^{14}$ & $-8,19 \cdot 10^{12}$ \\
\hline 5 & $-3,49 \cdot 10^{15}$ & $4,82 \cdot 10^{15}$ & $-8,99 \cdot 10^{12}$ & $-1,45 \cdot 10^{15}$ & $7,85 \cdot 10^{14}$ & $-9,15 \cdot 10^{14}$ & $8,96 \cdot 10^{13}$ & $-1,01 \cdot 10^{14}$ & $-1,16 \cdot 10^{16}$ & $1,98 \cdot 10^{14}$ \\
\hline 6 & $5,75 \cdot 10^{16}$ & $-7,96 \cdot 10^{16}$ & $3,15 \cdot 10^{14}$ & $2,39 \cdot 10^{16}$ & $-1,31 \cdot 10^{16}$ & $1,51 \cdot 10^{16}$ & $-1,48 \cdot 10^{15}$ & $1,68 \cdot 10^{15}$ & $2,15 \cdot 10^{17}$ & $-3,28 \cdot 10^{15}$ \\
\hline 7 & $-6,63 \cdot 10^{17}$ & $9,20 \cdot 10^{17}$ & $-5,67 \cdot 10^{15}$ & $-2,76 \cdot 10^{17}$ & $1,52 \cdot 10^{17}$ & $-1,75 \cdot 10^{17}$ & $1,71 \cdot 10^{16}$ & $-1,95 \cdot 10^{16}$ & $-2,83 \cdot 10^{18}$ & $3,79 \cdot 10^{16}$ \\
\hline 8 & $5,36 \cdot 10^{18}$ & $-7,46 \cdot 10^{18}$ & $6,32 \cdot 10^{16}$ & $2,24 \cdot 10^{18}$ & $-1,24 \cdot 10^{18}$ & $1,42 \cdot 10^{18}$ & $-1,38 \cdot 10^{17}$ & $1,59 \cdot 10^{17}$ & $2,70 \cdot 10^{19}$ & $-3,07 \cdot 10^{17}$ \\
\hline 9 & $-2,98 \cdot 10^{19}$ & $4,16 \cdot 10^{19}$ & $-4,53 \cdot 10^{17}$ & $-1,25 \cdot 10^{19}$ & $6,96 \cdot 10^{18}$ & $-7,92 \cdot 10^{18}$ & $7,71 \cdot 10^{17}$ & $-8,95 \cdot 10^{17}$ & $-1,85 \cdot 10^{20}$ & $1,72 \cdot 10^{18}$ \\
\hline 10 & $1,09 \cdot 10^{20}$ & $-1,53 \cdot 10^{20}$ & $2,04 \cdot 10^{18}$ & $4,58 \cdot 10^{19}$ & $-2,57 \cdot 10^{19}$ & $2,91 \cdot 10^{19}$ & $-2,83 \cdot 10^{18}$ & $3,31 \cdot 10^{18}$ & $8,87 \cdot 10^{20}$ & $-6,30 \cdot 10^{18}$ \\
\hline 11 & $-2,37 \cdot 10^{20}$ & $3,32 \cdot 10^{20}$ & $-5,27 \cdot 10^{18}$ & $-9,96 \cdot 10^{19}$ & $5,63 \cdot 10^{19}$ & $-6,32 \cdot 10^{19}$ & $6,14 \cdot 10^{18}$ & $-7,24 \cdot 10^{18}$ & $-2,84 \cdot 10^{21}$ & $1,37 \cdot 10^{19}$ \\
\hline 12 & $2,30 \cdot 10^{20}$ & $-3,25 \cdot 10^{20}$ & $5,99 \cdot 10^{18}$ & $9,73 \cdot 10^{19}$ & $-5,54 \cdot 10^{19}$ & $6,18 \cdot 10^{19}$ & $-5,99 \cdot 10^{18}$ & $7,13 \cdot 10^{18}$ & $5,45 \cdot 10^{21}$ & $-1,34 \cdot 10^{19}$ \\
\hline 13 & & & & & & & & & $-4,76 \cdot 10^{21}$ & \\
\hline
\end{tabular}


Table 3: The parameters An in the formula (5) for combined boundary conditions that is shown in the figure 1,c

\begin{tabular}{|c|c|c|c|c|c|c|c|c|c|c|}
\hline \multirow{2}{*}{$\mathrm{n}$} & \multicolumn{10}{|c|}{ The parameters a in the formula (4) } \\
\hline & a1 & a2 & a3 & a4 & a5 & a6 & a7 & a8 & a9 & a10 \\
\hline 1 & 年 & 170 & 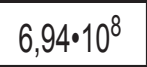 & $68 \cdot 10^{7}$ & $26 \cdot 10^{8}$ & $24 \cdot 10^{8}$ & $0<-10$ & $64 \cdot 10^{7}$ & $56 \cdot 10^{5}$ & $84 \cdot 10^{7}$ \\
\hline 2 & & & & | & & & & & & \\
\hline 3 & . & (1) & (1) & $36 \cdot 10^{11}$ & $15 \cdot 10^{12}$ & $20 \cdot 10^{12}$ & 14010 & 210 & $\angle .10$ & 0.10 \\
\hline 4 & (1) &, $73 \cdot 10^{13}$ & $46 \cdot 10^{14}$ & $36 \cdot 10^{13}$ & $7,99 \cdot 10^{13}$ & $4,48 \cdot 10^{13}$ & $-2,00 \cdot 10^{11}$ & $9,36 \cdot 10^{12}$ & $1,62 \cdot 10^{11}$ & $-6,58 \cdot 10^{12}$ \\
\hline 5 & $=0,10$ & $1,00 \%$ & $5,9 \angle{ }^{\circ} \mid 0$ & $-5,66 \cdot 10^{14}$ & $-1,92 \cdot 10^{15}$ & $-1,08 \cdot 10$ & $4,4 J 0$ & $2, \angle 5^{\circ} 10$ & $-3,53 \cdot 10^{12}$ & $1,00^{\circ}$ \\
\hline 6 & $1,01 \cdot 10^{17}$ & $05 \cdot 10^{16}$ & $71 \cdot 10^{16}$ & $27 \cdot 10^{15}$ & $3,14 \cdot 10^{16}$ & $1,79 \cdot 10^{16}$ & $-6,60 \cdot 10^{13}$ & $-3,68 \cdot 10^{15}$ & $5,14 \cdot 10^{13}$ & $-2,61 \cdot 10^{15}$ \\
\hline 7 & 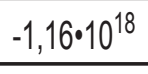 & $50 \cdot 10^{17}$ & $11 \cdot 10^{18}$ & $06 \cdot 10^{17}$ & $-3,59 \cdot 10^{17}$ & $-2,07 \cdot 10^{17}$ & $3 \cdot 10^{14}$ & $4,21 \cdot 10^{16}$ & $-5,09 \cdot 10^{14}$ & $3,00^{\circ}$ \\
\hline 8 & $9,30 \cdot 10^{18}$ & $-2,81 \cdot 10^{18}$ & $-8,94 \cdot 10^{18}$ & $8,49 \cdot 10^{17}$ & $2,88 \cdot 10^{18}$ & $1,68 \cdot 10^{18}$ & $-4,68 \cdot 10^{15}$ & $-3,38 \cdot 10^{17}$ & $3,41 \cdot 10^{15}$ & $-2,42 \cdot 10^{17}$ \\
\hline 9 & $-5,15 \cdot 10^{19}$ & 1,00010 & $4,94 \cdot 10^{19}$ & $-4,68 \cdot 10^{18}$ & $-1,59 \cdot 10^{19}$ & $-9,35 \cdot 10^{18}$ & $2,15 \cdot 10$ & $1,87 \cdot 10^{18}$ & $-1,48 \cdot 10^{16}$ & $1,34 \cdot 10^{18}$ \\
\hline 10 & $1,87 \cdot 1$ & $-5,65 \cdot 10^{19}$ & $-1,80 \cdot 10^{20}$ & 1,69 & $5,77 \cdot 10^{19}$ & $3,43 \cdot 10^{19}$ & $-6,12 \cdot 10^{16}$ & $-6,78 \cdot 10^{18}$ & $3,82 \cdot 10^{16}$ & $-4,89 \cdot 10^{18}$ \\
\hline 11 & $-4,04 \cdot 10^{20}$ & $1,22 \cdot 10^{20}$ & $3,87 \cdot 10^{20}$ & $-3,63 \cdot 10^{19}$ & $-1,24 \cdot 10^{20}$ & $-7,45 \cdot 10^{19}$ & $9,24 \cdot 10^{16}$ & $1,46 \cdot 10^{19}$ & $-4,70 \cdot 10^{16}$ & $1,06 \cdot 10^{19}$ \\
\hline 12 & $3,92 \cdot 10^{20}$ & $-1,18 \cdot 10^{20}$ & $-3,74 \cdot 10^{20}$ & $3,50 \cdot 10^{19}$ & $1,20 \cdot 10^{20}$ & $7,28 \cdot 10^{19}$ & $-4,90 \cdot 10^{16}$ & $-1,41 \cdot 10^{19}$ & $9,47 \cdot 10^{15}$ & $-1,03 \cdot 10^{19}$ \\
\hline
\end{tabular}

Table 4: The parameters An in the formula (5) for combined boundary conditions that is shown in the figure 1,d

\begin{tabular}{|c|c|c|c|c|c|c|c|c|c|c|}
\hline \multirow{2}{*}{$n(5)$} & \multicolumn{10}{|c|}{ The parameters a in the formula (4) } \\
\hline & a1 & a2 & a3 & a4 & a5 & a6 & a7 & a8 & a9 & a10 \\
\hline 1 & $-8,37 \cdot 10^{7}$ & $1,18 \cdot 10^{8}$ & $1,51 \cdot 10^{8}$ & $2,79 \cdot 10^{8}$ & $1,77 \cdot 10^{8}$ & $-4,83 \cdot 10^{8}$ & $-5,58 \cdot 10^{7}$ & $-2,86 \cdot 10^{7}$ & $2,57 \cdot 10^{7}$ & $-1,46 \cdot 10^{9}$ \\
\hline 2 & $1,22 \cdot 10^{10}$ & $-1,72 \cdot 10^{10}$ & $-2,26 \cdot 10^{10}$ & $-4,16 \cdot 10^{10}$ & $-2,63 \cdot 10^{10}$ & $7,20 \cdot 10^{10}$ & $8,34 \cdot 10^{9}$ & $4,27 \cdot 10^{9}$ & $-3,82 \cdot 10^{9}$ & $2,30 \cdot 10^{11}$ \\
\hline 3 & $-7,70 \cdot 10^{11}$ & $1,09 \cdot 10^{12}$ & $1,46 \cdot 10^{12}$ & $2,70 \cdot 10^{12}$ & $1,70 \cdot 10^{12}$ & $-4,66 \cdot 10^{12}$ & $-5,40 \cdot 10^{11}$ & $-2,76 \cdot 10^{11}$ & $2,46 \cdot 10^{11}$ & $-1,59 \cdot 10^{13}$ \\
\hline 4 & $2,79 \cdot 10^{13}$ & $-3,94 \cdot 10^{13}$ & $-5,46 \cdot 10^{13}$ & $-1,01 \cdot 10^{14}$ & $-6,37 \cdot 10^{13}$ & $1,74 \cdot 10^{14}$ & $2,02 \cdot 10^{13}$ & $1,03 \cdot 10^{13}$ & $-9,19 \cdot 10^{12}$ & $6,46 \cdot 10^{14}$ \\
\hline 5 & $-6,53 \cdot 10^{14}$ & $9,19 \cdot 10^{14}$ & $1,32 \cdot 10^{15}$ & $2,45 \cdot 10^{15}$ & $1,54 \cdot 10^{15}$ & $-4,20 \cdot 10^{15}$ & $-4,89 \cdot 10^{14}$ & $-2,49 \cdot 10^{14}$ & $2,21 \cdot 10^{14}$ & $-1,72 \cdot 10^{16}$ \\
\hline 6 & $1,04 \cdot 10^{16}$ & $-1,46 \cdot 10^{16}$ & $-2,18 \cdot 10^{16}$ & $-4,06 \cdot 10^{16}$ & $-2,54 \cdot 10^{16}$ & $6,93 \cdot 10^{16}$ & $8,09 \cdot 10^{15}$ & $4,12 \cdot 10^{15}$ & $-3,64 \cdot 10^{15}$ & $3,19 \cdot 10^{17}$ \\
\hline 7 & $-1,15 \cdot 10^{17}$ & $1,61 \cdot 10^{17}$ & $2,52 \cdot 10^{17}$ & $4,71 \cdot 10^{17}$ & $2,94 \cdot 10^{17}$ & $-8,00 \cdot 10^{17}$ & $-9,35 \cdot 10^{16}$ & $-4,75 \cdot 10^{16}$ & $4,19 \cdot 10^{16}$ & $-4,22 \cdot 10^{18}$ \\
\hline 8 & $8,86 \cdot 10^{17}$ & $-1,25 \cdot 10^{18}$ & $-2,04 \cdot 10^{18}$ & $-3,83 \cdot 10^{18}$ & $-2,38 \cdot 10^{18}$ & $6,47 \cdot 10^{18}$ & $7,58 \cdot 10^{17}$ & $3,84 \cdot 10^{17}$ & $-3,38 \cdot 10^{17}$ & $4,04 \cdot 10^{19}$ \\
\hline 9 & $-4,69 \cdot 10^{18}$ & $6,60 \cdot 10^{18}$ & $1,14 \cdot 10^{19}$ & $2,14 \cdot 10^{19}$ & $1,33 \cdot 10^{19}$ & $-3,60 \cdot 10^{19}$ & $-4,24 \cdot 10^{18}$ & $-2,14 \cdot 10^{18}$ & $1,88 \cdot 10^{18}$ & $-2,77 \cdot 10^{20}$ \\
\hline 10 & $1,62 \cdot 10^{19}$ & $-2,29 \cdot 10^{19}$ & $-4,17 \cdot 10^{19}$ & $-7,88 \cdot 10^{19}$ & $-4,86 \cdot 10^{19}$ & $1,32 \cdot 10^{20}$ & $1,56 \cdot 10^{19}$ & $7,85 \cdot 10^{18}$ & $-6,85 \cdot 10^{18}$ & $1,34 \cdot 10^{21}$ \\
\hline 11 & $-3,31 \cdot 10^{19}$ & $4,66 \cdot 10^{19}$ & $9,05 \cdot 10^{19}$ & $1,72 \cdot 10^{20}$ & $1,06 \cdot 10^{20}$ & $-2,86 \cdot 10^{20}$ & $-3,38 \cdot 10^{19}$ & $-1,70 \cdot 10^{19}$ & $1,48 \cdot 10^{19}$ & $-4,30 \cdot 10^{21}$ \\
\hline 12 & $3,01 \cdot 10^{19}$ & $-4,24 \cdot 10^{19}$ & $-8,83 \cdot 10^{19}$ & $-1,69 \cdot 10^{20}$ & $-1,03 \cdot 10^{20}$ & $2,79 \cdot 10^{20}$ & $3,31 \cdot 10^{19}$ & $1,66 \cdot 10^{19}$ & $-1,44 \cdot 10^{19}$ & $8,31 \cdot 10^{21}$ \\
\hline 13 & & & & & & & & & & $-7,28 \cdot 10^{21}$ \\
\hline
\end{tabular}

Substituting reference parameters An from the table 1 to formula (4) with respect to (5) we obtain critical force of critical force to plate in the form of a rectangular. Comparing FFIM results with the FEM we obtain the following results:
- for A scheme error not exceed $2.99 \%$;

- for B scheme error not exceed $2.93 \%$;

- for C scheme error not exceed $2.12 \%$;

- for D scheme error not exceed $3.31 \%$. 


\section{CONCLUSION}

Summarizing the results it can be obtained the following conclusions:

The form factor interpolation method is shared to stability analysis of elastic orthotropic rectangular plates the opposite sides of which are simply supported and two other sides are clamped or simply supported.

The functional relationships of critical force, form factor and flexural rigidity ratios are obtained

The researching results are compared with the FEM and demonstrate good accuracy

Proposed methodic can be extended to stability analysis of plates with other form of the midplane, boundary conditions and loadings.

\section{REFERENCES}

1) Timoshenko S.P., (1971). Ustojchivost' sterzhnej, plastin i obolochek [Stability of rods, plates and shells]. Moscow: Nauka Publ.

2) Vol'mirA.S., (1971). Ustojchivost' deformiruemyh sistem [Stability of deformable systems]. Moscow: Nauka Publ..

3) Dmitrienko Yu.I., (2015). Teoriya ustoychivosti plastin, osnovannaya na asimptoticheskom analize uravneniy teorii ustoychivosti trekhmernykh uprugikh sred [Stability theory of plates, based on the asymptotic analysis of the equations of the theory of stability of three-dimensional elastic medium]. Nauka i innovacii, 9(45), 1-26.

4) Belous A.A. \& Belous V.A., (1977). Ustojchivost' prjamougol'nyh plastin za predelom uprugosti $s$ uchetom szhimaemosti materiala [Stability of rectangular plates beyond the elastic limit, taking into account the compressibility of the material]. Uchenye zapiski CAGI, 6(8), 107-118.

5) Annenkov L.V., (2015). Issledovanie ustojchivosti zashhemlennoj prjamougol'noj plastiny, szhatoj v odnom napravlenii [Stability analysis of rectangular plate, clamped along the contour and compressed in on direction]. Vestnik Gosudarstvennogo universiteta morskogo i rechnogo flota im. Admirala S.O., 3, 48-53.

6) Lopatin A.V. \& Avakumov R.V., Ustojchivost' ortotropnoj plastiny s dvumja svobodnymikrajami, nagruzhennoj izgibajushhim momentom $v$ ploskosti [Stability of an orthotropic plate with two Svobodnaya loaded by bend- ing moment in the plane]. U: Reshetnevskie chtenija, 2009, Krasnoyarsk. 35-36.

7) Ropalin Siahaan, Poologanathan Keerthan \& Mahen Mahendran, (2016). Finite element modeling of rivet fastened rectangular hollow flange channel beams subject to local buckling. Engineering Structures, 126, 311-327. doi:10.1016/j.engstruct.2016.07.004

8) Wael F. Ragheb, (2016). Estimating the local buckling capacity of structural steel I-section columns at elevated temperatures. ThinWalled Structures, 107, 18-27. doi:10.1016/ j.tws.2016.05.016

9) Angus C.C. Lam, Yanyang Zhang, Yi Qin, Michael C.H. Yam \& V.P. Iu, (2016). Design for inelastic local web buckling of coped beams. Journal of Constructional Steel Research, 125, 173-189. doi:10.1016/ j.jcsr.2016.06.016

10) Korobko A.V., (1999). Geometricheskoe modelirovanie formy oblasti $v$ dvumernyh zadachah teorii uprugosti [Geometric modeling of area shape in two-dimensional problems of the elasticity theory]. Moscow: ASV Publ..

11) Korobko V.I., Korobko A.V., Chernyaev A.A. \& Savin S.Yu., (2015). Determination of maximum deflection at cross bending parallelogram plates using conformal radius ratio interpolation technique. Journal of the Serbian Society for Computational Mechanics, 9(2), 36-45. doi:10.5937/jsscm1501036K

12) Korobko A.V. \& Chikulaev A.V., (2006). Raschet ustojchivosti prjamougol'noj $v$ plane pologoj obolochki metodom interpoljacii po kojefficientu formy [Stability analysis of rectangular in the plane shallow shell, applying the form factor interpolation method]. Izvestiya Orlovskogo Gosudarstvennogo Tehnicheskogo Universiteta. Seriya: Stroitel'stvo i transport, 3(4), 36-39.

13) Korobko V., Korobko A. \& Chernyaev A., (2016). Isoperimetric properties of the torsion rigidity of convex sections. Procedia Engineering, 150, 1648-1656. doi:10.1016/ j.proeng.2016.07.146

14) Korobko V.I., Korobko A.V., Savin S.Yu. \& Chernyaev A.A., (2016). Solving the transverse bending problem of thin elastic orthotropic plates with form factor interpolation method. Journal of the Serbian Society for Computational Mechanics, 2(10), 9-17. doi:10.5937/jsscm1602009K 
15) Korobko A.V., Savin S.Yu. \& Filatova S.A., (2016). Determination of stiffness and fundamental frequency of oscillations of fixed circuit plates. Izvestiya Vysshikh Uchebnykh Zavedenii, Seriya Teknologiya Tekstil'noi Promyshlennosti, 3(363), 290-295.

16) Korobko V.I. \& Korobko A.V., (2010). Stroitel'naja mehanika plastinok: Tehniches- kaja teorija [Structural mechanics of plates: Technical theory]. Moscow: Izdatel'skij dom "Spektr" Publ.

Paper sent to revision: 20.07.2017.

Paper ready for publication: 15.09.2017. 